\title{
Mes tatouages ont causé ma sécheresse oculaire?
}

\section{Un nouveau regard sur le diagnostic et le traitement pour les patientes ayant un tatouage (maquillage permanent) de contour des yeux}

Caitlin J. Morrison, DO

Joseph M. Stamm, DO, FAAO (Dip)

SUNY College of Optometry, New York, NY, États-Unis

cmorrison@sunyopt.edu

\begin{abstract}
Résumé
But. Le présent rapport de cas décrit les répercussions potentielles d'un tatouage de contour des yeux sur la structure et le fonctionnement des paupières, soit des symptômes accrus de sécheresse oculaire et d'autres anomalies.

Rapport de cas. Une femme de 59 ans d'origine hispanique, aux prises depuis longtemps avec des symptômes de sécheresse oculaire peu soulagés par des larmes artificielles, a consulté pour une évaluation. Les examens d'imagerie ont révélé une perte de glandes de Meibomius, probablement causée par le tatouage de contour des yeux. L'application de compresses chaudes, le massage palpébral et l'utilisation de larmes artificielles à base de lipides ont permis d'atténuer les symptômes et d'améliorer les mesures objectives.

Conclusions. Un contour des yeux tatoué de façon permanente peut accroître la sécheresse oculaire par deux mécanismes principaux : perturbation de l'architecture des paupières et inflammation chronique due aux granules de pigments dans l'encre de tatouage. Reconnaître ces possibles effets chez les patientes ayant le contour des yeux tatoué peut aider à offrir un traitement adapté à l'étiologie de la sécheresse oculaire : stimuler les glandes de Meibomius restantes par des compresses chaudes et un massage palpébral, et utiliser des larmes artificielles à base de lipides pour pallier l'absence de sécrétion de lipides par les glandes de Meibomius qui manquent.
\end{abstract}

\section{Introduction}

La sécheresse oculaire touche de $5 \%$ à $34 \%$ (prévalence) de la population mondiale et se divise en deux sous-groupes, selon l'étiologie : par déficience aqueuse et par hyperévaporation ${ }^{1}$. La sécheresse oculaire par déficience aqueuse apparaît lorsque les glandes lacrymales ne produisent pas suffisamment de larmes aqueuses pour lubrifier l'œil. Elle ne représente qu'environ $10 \%$ des cas de sécheresse oculaire. La forme la plus courante, qui fait l'objet de ce rapport de cas, est la sécheresse oculaire par hyperévaporation. Elle est due à un dysfonctionnement des glandes de Meibomius, lequel entraîne une sous-sécrétion de la couche lipidique nécessaire au maintien des larmes aqueuses sur les yeux. La forme hyperévaporation et les formes mixtes hyperévaporation et déficience aqueuse représentent environ $80 \%$ des cas de sécheresse oculaire.

\section{Rapport De Cas}

Une femme hispanique de 59 ans a été adressée pour l'évaluation d'une sécheresse oculaire, à la demande de son professionnel de première ligne pour les soins de la vue. Elle avait des symptômes de sécheresse de longue date, un léger prurit oculaire associé à des allergies au pollen et des sensations de légères brûlures oculaires. Une période prolongée devant la télévision ou l'ordinateur provoquait un larmoiement, mais sans écoulement de larmes sur les joues. La durée des symptômes était inconnue. La patiente utilisait des gouttes ophtalmiques de kétotifène deux fois par jour et les larmes artificielles Systane Ultra (Alcon Laboratories, Inc), deux fois par jour dans les deux yeux. Elle n'avait jamais utilisé de compresses chaudes et n'avait jamais eu de recommandation en ce sens. Elle s'était fait appliquer un contour des yeux 

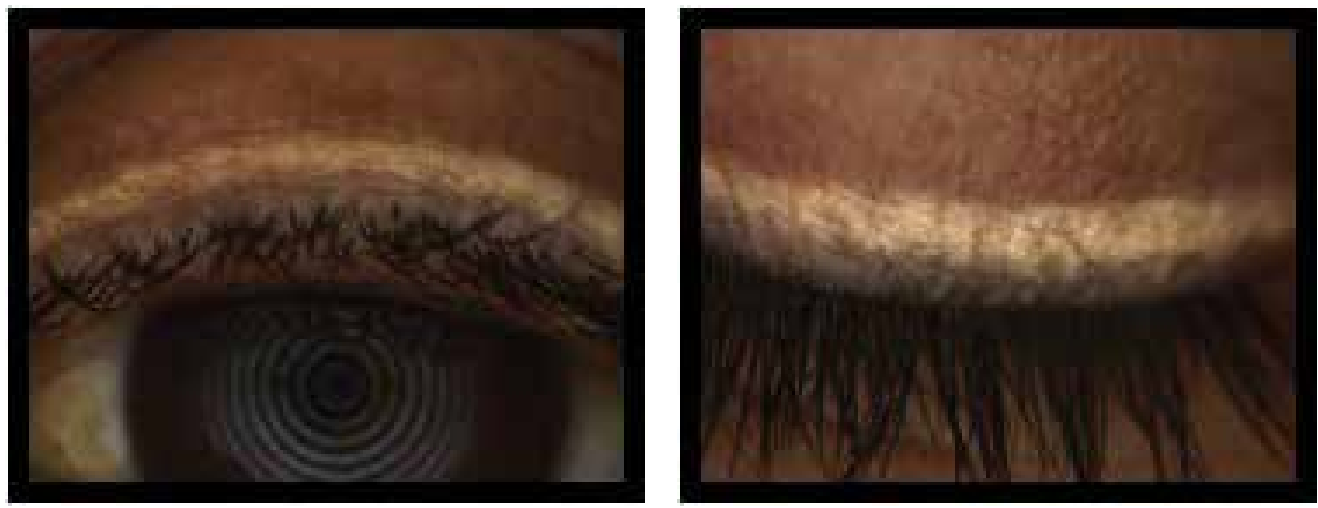

Figure 1. Contour des yeux tatoué sur les paupières supérieures
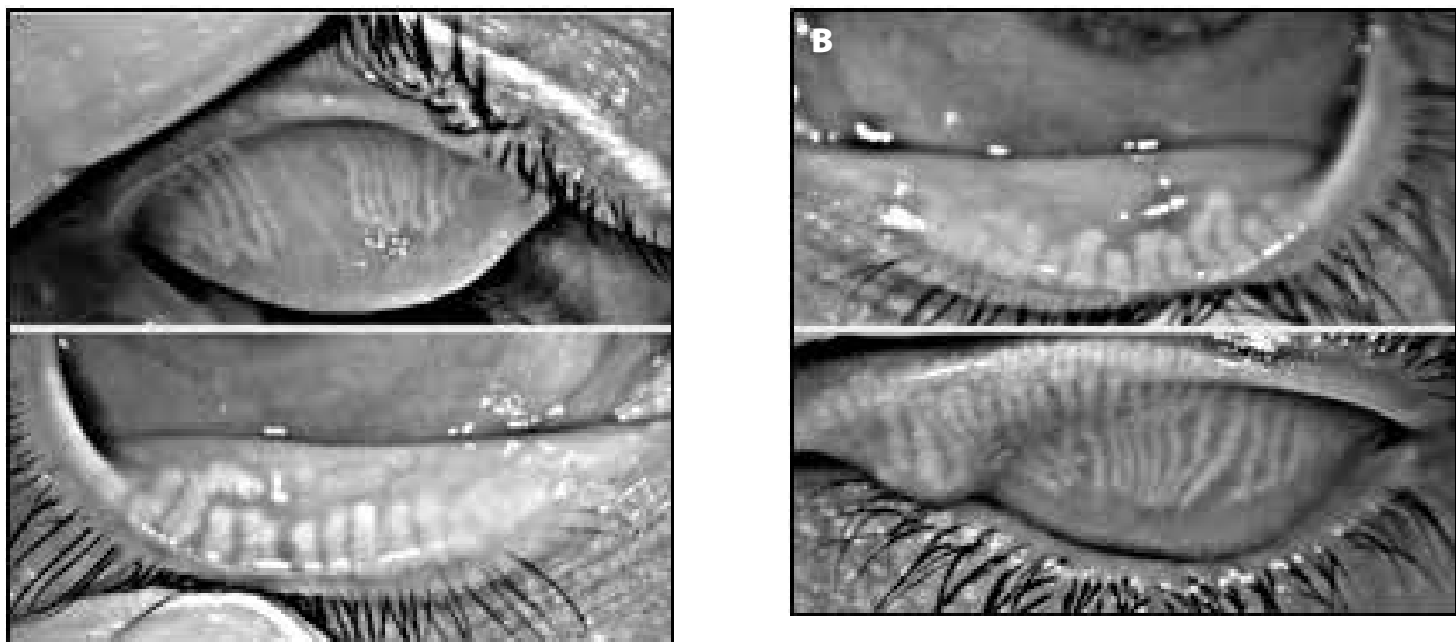

Figure 2. Meibographie prise avec un kératographe 5M d'Oculus (Oculus, Inc.).

a.) Eil droit: Les flèches indiquent une perte de glandes de Meibomius (paupière supérieure) et un raccourcissement des glandes de Meibomius (paupière inférieure).

b.) Eil gauche : Les flèches indiquent un raccourcissement des glandes de Meibomius (paupières supérieure et inférieure).

permanent sur les paupières supérieures (encre jaune) et inférieures (encre noire) quelque 25 ans plus tôt; celui des paupières inférieures n'était toutefois pas visible, s'étant dissous lorsqu'elle avait utilisé une crème blanchissante pour la peau. Elle n'avait jamais porté de lentilles cornéennes. Elle avait reçu un diagnostic de diabète de type 2 environ 4 mois auparavant, ne connaissait pas sa glycémie et prenait 3 comprimés de $500 \mathrm{mg}$ de metformine par voie orale, deux fois par jour.

Au cours de l'examen initial, les observations pertinentes comprenaient une acuité visuelle corrigée de 20/20 à distance et de près. Pendant l'examen à la lampe à fentes, un contour des yeux tatoué a été noté sur la paupière supérieure des deux yeux (photo 1). Un léger dysfonctionnement des glandes de
Meibomius a été détecté, accompagné de glandes aux orifices bouchés et de sécrétions turbides exprimées par pression digitale dans les deux yeux. La partie inférieure des deux cornées a pris une coloration ponctuée superficielle avec la fluorescéine sodique. Le temps de rupture du film lacrymal (TRFL) mesuré par le praticien a été de 2 secondes dans chaque œil. Le vert de lissamine s'est fixé seulement à des pinguéculas du côté temporal et nasal dans les deux yeux. Le test de Jones I, effectué en raison de ses symptômes de larmoiement, a démontré que les canaux lacrymaux et le système de drainage lacrymal étaient perméables dans les deux yeux.

Un kératographe $5 \mathrm{M}$ d'Oculus (Oculus, Inc.) a servi à quantifier la sécheresse oculaire par la mesure objective d'un TRFL. Dans l'œil droit, la valeur dans la zone centrale de la 

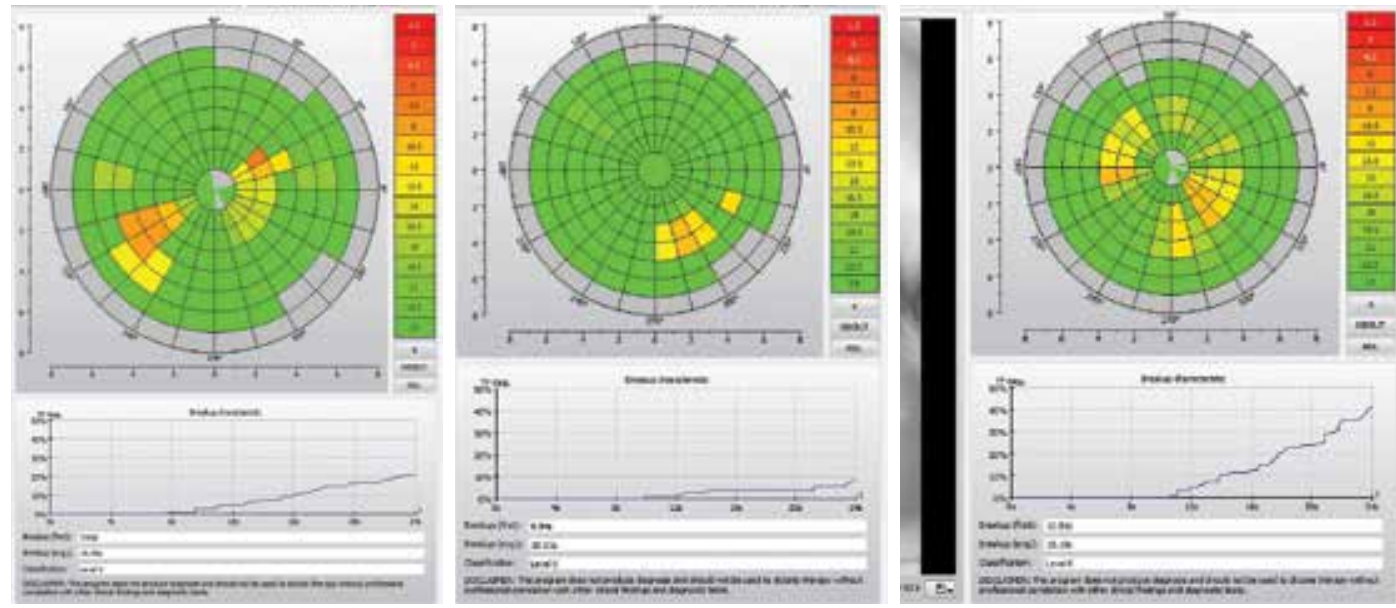

Figure 3. Temps de rupture du film lacrymal mesuré au kératographe (Oculus, Inc.).

Eil droit (de gauche à droite) : examen initial, deuxième examen de suivi, troisième examen de suivi.
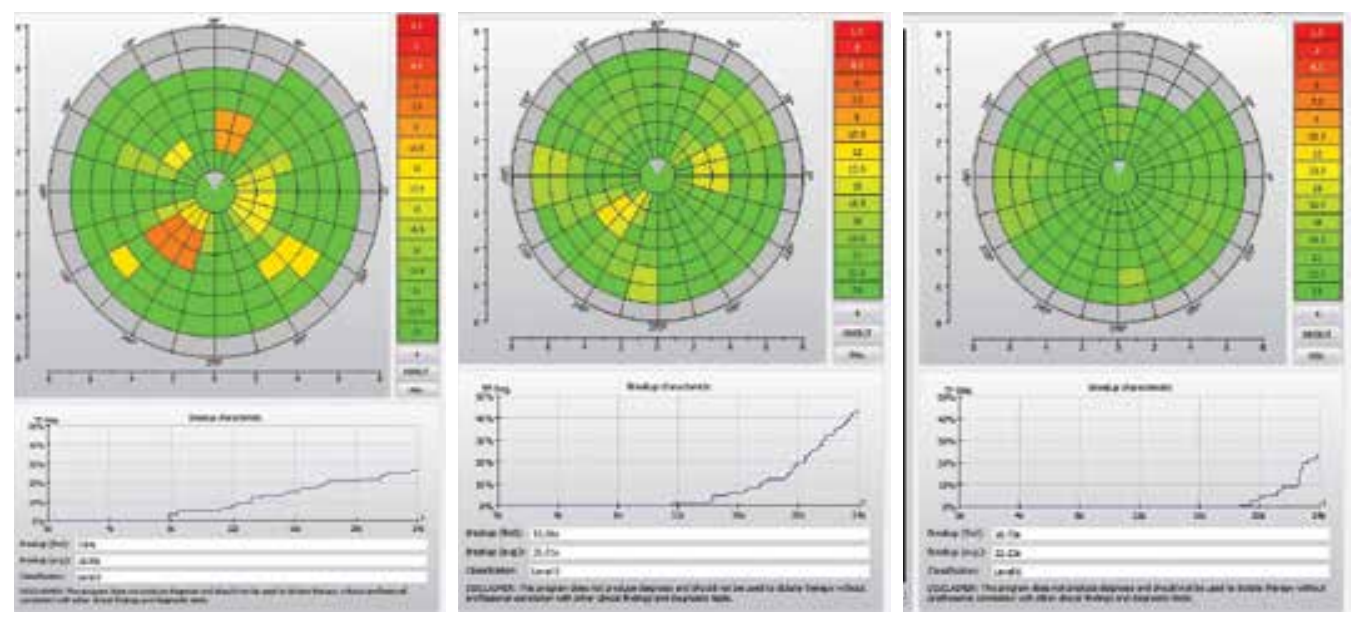

Figure 4. Temps de rupture du film lacrymal mesuré au kératographe (Oculus, Inc.).

Eil gauche (de gauche à droite) : examen initial, deuxième examen de suivi, troisième examen de suivi.

cornée a été de 7,65 secondes et la moyenne pour la cornée entière, de 16,55 secondes (photo 3). Dans l'œil gauche, la valeur dans la zone centrale de la cornée a été de 7,84 secondes et la moyenne pour la cornée entière, de 15,05 secondes (photo 4). La structure physique des glandes de Meibomius a été révélée par les images de meibographie infrarouge recueillies avec le kératographe. Les images de l'œil droit montraient une région distincte de perte des glandes de Meibomius dans la paupière supérieure, et un raccourcissement des glandes de Meibomius encore présentes sur les paupières supérieure et inférieure. Les images de l'œil gauche ont aussi mis en évidence un raccourcissement des glandes de Meibomius sur les paupières supérieure et inférieure (photo 2).
À la lumière des résultats, un diagnostic de dysfonctionnement des glandes de Meibomius a été posé. Le contour des yeux tatoué a été un facteur causal présumé dans l'étiologie de la maladie. La première stratégie de traitement a consisté à soutenir le fonctionnement des glandes de Meibomius restantes par l'ajout de compresses chaudes et d'un massage palpébral, trois fois par jour, et l'utilisation de larmes artificielles Systane Ultra quatre fois plutôt que deux fois par jour, dans chaque œil.

À une visite de suivi deux semaines plus tard, la patiente a indiqué que ses symptômes de sécheresse et les sensations de brûlure avaient diminué. Elle a dit avoir une excellente observance de toutes les mesures de traitement de la sécheresse oculaire. Quantitativement, le TRFL dans la zone centrale de 
la cornée avec le kératographe 5M a augmenté de 29,9\% dans l'œil droit, passant à 9,94 secondes. Le TRFL dans l'œil gauche a été de 11,60 secondes, en hausse de 47,9\%. Le TRFL mesuré par le praticien a été amélioré de 2 à 3 secondes dans les deux yeux. La coloration de la cornée par la fluorescéine sodique et de la conjonctive par le vert de lissamine a été réduite dans les deux yeux.

Pour mieux rétablir la couche lipidique des larmes, il a été décidé de remplacer Systane Ultra, des larmes artificielles à base d'eau, par Systane Balance (Alcon Laboratories, Inc), des larmes artificielles à base de lipides. Une étude a montré que Systane Balance avait amélioré le TRFL de $33 \%$ chez 49 patients atteints d'un dysfonctionnement des glandes de Meibomius, par rapport au traitement habituel de la sécheresse oculaire ${ }^{2}$.

Deux mois plus tard, au deuxième examen de suivi, la patiente dit avoir obtenu un soulagement plus grand avec Systane Balance qu'avec Systane Ultra. Par rapport à sa valeur initiale, le TRFL mesuré avec le kératographe $5 \mathrm{M}$ s'est amélioré de 34,7 \% (10,31 secondes) dans l'œil droit et de 138,7\% (18,72 secondes) dans l'œil gauche. À l'examen effectué par le praticien, l'augmentation du TRFL par rapport à la valeur initiale a été de 2 à 5 secondes dans l'œil droit et de 2 à 7 secondes dans l'œil gauche. Avec la fluorescéine sodique, la coloration ponctuée superficielle a été infime dans le bas de l'œil droit, et totalement absente dans le bas de l'œil gauche. La coloration par le vert de lissamine est restée la même que lors du premier rendez-vous de suivi.

La réponse au traitement a confirmé le diagnostic de dysfonctionnement des glandes de Meibomius dans les deux yeux. Notre patiente a été informée qu'il s'agissait d'une maladie chronique exigeant une prise en charge à long terme, et qu'il lui fallait continuer le traitement par des compresses chaudes et un massage palpébral, trois fois par jour, et par les larmes artificielles Systane Balance (Alcon Laboratories, Inc.), quatre fois par jour. On lui a demandé de revenir à la clinique six mois plus tard pour son examen annuel complet de la vue.

\section{Discussion}

Le dysfonctionnement des glandes de Meibomius se caractérise par une obstruction des canaux terminaux et par des changements qualitatifs et quantitatifs dans les sécrétions des glandes de Meibomius ${ }^{3}$. Chez une patiente ayant un contour des yeux tatoué de façon permanente, les deux principales causes d'une sécheresse oculaire accrue seraient, en théorie, la perturbation de l'architecture des paupières durant l'intervention de tatouage et la réaction inflammatoire du corps aux molécules de pigment contenues dans l'encre. Comme
$42,4 \%$ des chirurgiens plasticiens offrent l'intervention de maquillage permanent, il est important que les professionnels des soins de la vue tiennent compte des effets de celle-ci sur la paupière lorsqu'ils examinent des patientes ${ }^{4}$.

L'anatomie de la paupière, de son bord antérieur à son extrémité postérieure, comprend l'épiderme, le derme, le muscle orbiculaire et la plaque tarsale. Les glandes de Meibomius se trouvent dans la plaque tarsale. L'aiguille de tatouage n'est censée placer le pigment que dans le derme antérieur, mais il est possible que l'aiguille et le pigment pénètrent plus profondément que prévu ${ }^{5}$. Si l'intervention n'est pas effectuée correctement, l'aiguille peut entrer assez loin pour perturber la zone qui contient les glandes de Meibomius. L'inflammation induite par ce type de trauma pourrait endommager les glandes, et ainsi les tronquer ou les atrophier ${ }^{6,7}$. Des rapports font état d'une aiguille de tatouage introduite assez profondément pour lacérer la paupière et placer l'encre de tatouage dans la conjonctive bulbaire ${ }^{6}$. Ces rapports constituent d'autres preuves que l'architecture de la paupière, dont les glandes de Meibomius, peut être altérée à la fois par l'aiguille de tatouage et par le pigment.

Un tatouage de contour des yeux peut exacerber la sécheresse oculaire par un deuxième mécanisme, soit la réaction des particules d'encre avec les tissus palpébraux, laquelle est susceptible de causer une légère inflammation chronique $^{1}$. Cette inflammation peut abîmer les structures qui produisent les couches du film lacrymal, telles que les glandes lacrymales accessoires de Wolfring et les glandes de Meibomius. Les glandes de Wolfring, qui se trouvent dans la conjonctive palpébrale et ressemblent aux glandes lacrymales principales, sécrètent des électrolytes, des liquides et des protéines qui lubrifient la surface oculaire ${ }^{8}$. Les glandes de Meibomius produisent une substance lipidique qui agit comme une barrière à l'évaporation entre les larmes aqueuses et le milieu extérieur?

Il a été démontré que l'encre de tatouage renferme des nanoparticules de carbone ainsi que d'autres additifs et de $l^{\prime} e^{10}{ }^{10}$. Ces nanoparticules peuvent déclencher la formation, dans la peau, d'espèces réactives de l'oxygène qui causent l'inflammation. Une inflammation chronique focale, sans granulomes, a été notée au cours de l'analyse histopathologique d'échantillons prélevés sur la paupière après le tatouage ${ }^{11}$. Des fibroblastes dermiques chargés de pigment granulaire noir ont aussi été observés en histologie. Les composés chimiques présents dans un échantillon d'encre noire sont notamment l'arsenic et la p-phénylènediamine, pour lesquels des réactions ont été signalées, en particulier l'hypomélanose dans la peau. 
Aux États-Unis, la composition de l'encre de tatouage est réglementée par la Food and Drug Administration, mais la pratique du tatouage comme telle ne l'est pas ${ }^{5}$. Par conséquent, bon nombre des pigments utilisés ne sont pas approuvés pour un contact avec la peau, et aucun pigment n'est approuvé pour une injection ou une implantation dans la peau. Lorsqu'un pigment d'encre de tatouage est placé à un endroit, il est possible que l'encre ait un effet d'étalement et s'infiltre dans plusieurs couches de la paupière ${ }^{12}$. Des changements chimiques risquent donc de survenir dans des tissus qui n'étaient pas visés. Par ailleurs, des réactions granulomateuses allergiques aux particules d'encre peuvent se manifester, sous forme de sensibilité, d'enflure, de démangeaisons et de bosses ${ }^{13,14}$. Les auteurs d'une étude ont réalisé des entrevues chez 92 patientes ayant eu des réactions indésirables à des interventions de maquillage permanent. Au moment de l'entrevue, $68 \%$ de ces patientes présentaient des réactions non résolues (durée des symptômes allant de 5,5 mois à 3 ans), ce qui montre la possible chronicité de la réaction inflammatoire ${ }^{13}$.

Selon une étude publiée récemment, un tatouage de contour des yeux est directement lié à une perte de glandes de Meibomius et à une instabilité du film lacrymal' ${ }^{3}$. L'étude a été menée chez 40 femmes, 10 qui avaient un contour des yeux tatoué, et 30 qui n'en avaient pas (groupe témoin). Les résultats de l'étude, statistiquement significatifs, ont confirmé qu'un contour des yeux tatoué était associé à une érosion cornéenne plus étendue, à un volume de sécrétions lacrymales moindre, à un TRFL plus court et à une perte accrue de glandes de Meibomius.

Chez notre patiente, les compresses chaudes et le massage palpébral ont permis d'augmenter la sécrétion de la couche lipidique du film lacrymal par les glandes de Meibomius fonctionnelles qui restaient. Des larmes artificielles à base de lipides ont été ajoutées pour suppléer à l'aspect lipidique du meibum que les glandes manquantes ne pouvaient produire. Ces deux modalités de traitement ont rendu le film lacrymal plus stable, atténué les symptômes de sécheresse oculaire et amélioré les mesures cliniques objectives. Notre patiente aurait peut-être présenté un dysfonctionnement des glandes de Meibomius et des symptômes de sécheresse oculaire si elle n'avait pas eu le contour des yeux tatoué. Toutefois, bien que la littérature à ce sujet soit limitée, nous ne pouvons exclure un lien causal entre le contour des yeux tatoué et la perte de glandes de Meibomius dans son cas. Comme il s'agit d'une intervention courante chez les femmes, il est important de tenir compte du contour des yeux tatoué et de ses effets potentiels sur les glandes de Meibomius dans l'évaluation de l'étiologie de la sécheresse oculaire chez une patiente.

\section{Remerciements/divulgations}

Les auteurs n'ont aucune déclaration d'intérêts à signaler.

\section{Références}

1. Messmer EM. The pathophysiology, diagnosis, and treatment of dry eye disease. Dtsch Arztebl Int. 2015 [cité le ler octobre 2015];112(5):71-81. doi : 10.3238/arztebl.2015.0071

2. Benelli U. Systane lubricant eye drops in the management of ocular dryness. Clin Ophthalmol. 2011 [cité le ler octobre 2015]; 5:783-90. doi : 10.2147/ OPTH.S13773.

3. Lee YB, Kim JJ, Hyon JY, et al. Eyelid Tattooing Induces Meibomian Gland Loss and Tear Film Instability". Cornea [Internet]. 2015 [cité le 1er octobre 2015]; 34(7):750-5. doi : 10.1097/ICO.0000000000000452.

4. Kojima T, Dogru M, Matsumoto Y, et al. Tear Film and Ocular Surface Abnormalities After Eyelid Tattooing. Ophthal Plast Reconstr Surg. 2005 [cité le 1er octobre 2015]; 21(1):69-71. doi: 10.1097/01. IOP.0000153028.08506.47.

5. De Cuyper C. Permanent makeup: indications and complications. Clin Dermatol. 2008 [cité le ler octobre 2015]; 26(1):30-4. doi : 10.1016/j. clindermatol.2007.10.009.

6. De M, Marshak H, Uzcategui N, et al. Full-thickness eyelid penetration during cosmetic blepharopigmentation causing eye injury. J Cosmet Dermatol. 2008 [cité le 1er octobre 2015]; 7(1):35-8. doi : 10.1111/j.14732165.2008.00340.x.

7. Goldberg RA, Shorr N. Complications of blepharopigmentation. Ophthalmic Surg. 1989 [cité le 1er octobre 2015]; 20(6):420-3.

8. Ubels JL, Gipson IK, Spurr-Michaud SJ, et al. Gene expression in human accessory lacrimal glands of Wolfring. Invest Ophthalmol Vis Sci. 2012 [cité le 1er octobre 2015]; 53(11):6738-47. doi : 10.1167/iovs.12-10750.

9. McCulley JP, Shine WE. Meibomian gland function and the tear lipid layer. Ocul Surf. 2003 [cité le ler octobre 2015]; 1(3):97-106. http://dx.doi. org/10.1016/S1542-0124(12)70138-6.

10. Goldman A, Wollina U. Severe unexpected adverse effects after permanent eye makeup and their management by Q-switched Nd:YAG laser. Clin Interv Aging. 2014 [cité le ler octobre 2015]; 11(9):1305-9. doi : 10.2147/CIA. S67167.

11. Liao JC, Proia AD, Ely PH, et al. Late-onset melanopenic hypomelanosis as a complication of cosmetic eyeliner tattoo. J Am Acad Dermatol. 2013 p. [cité le 1er octobre 2015]; 69(3):144-6. doi : 10.1016/j.jaad.2013.02.029.

12. Peters NT, Conn H, Cote MA. Extensive lower eyelid pigment spread after blepharopigmentation. Ophthal Plast Reconstr Surg. 1999 [cité le $1^{\text {er }}$ octobre 2015]; 15(6):445-7. doi: 10.1097/00002341-199911000-00016.

13. Straetemans M, Katz L, Belson M. Adverse Reactions after PermanentMakeup Procedures. N Engl J Med. 2007 [cité le 25 août 2015]; 356:2753. doi : 10.1056/NEJMc063122.

14. Vagefi MR, Dragan L, Hughes SM, et al. Adverse reactions to permanent eyeliner tattoo. Ophthal Plast Reconstr Surg. 2006 [cité le $1^{\text {er }}$ octobre 2015]; 22(1):48-51. doi : 10.1097/01.iop.0000196713.94608.29. 